\title{
The evolution of outpatient parenteral antibiotic therapy
}

\author{
Sanjay Valabh Patel
}

Managing children on intravenous antibiotics (IVABs) at home is not new. The origins of outpatient parenteral antibiotic therapy (OPAT) can be traced back to the 1970s, initially for children with cystic fibrosis. ${ }^{1}$ Managing children at home offers clear benefits in terms of parent and patient satisfaction, psychological well-being, return to school/employment and reductions in healthcare-associated infections; as a result, children are being routinely ambulated on IVABs from paediatric units in Europe and elsewhere. However, there has been a recent resurgence of interest in this area, driven in part by the severe financial constraints being faced by hospitals, making OPAT an attractive option for reducing operational costs through reduced inpatient stays. The British Society of Antimicrobial Chemotherapy (BSAC) has recently published paediatric OPAT guidelines. ${ }^{2}$ The focus of these recommendations, as well as the majority of the research literature, has been the management of children with complex infections requiring prolonged courses of IVABs.

Hodgson et $a l^{3}$ describe a large prospective cohort of children managed within an OPAT service in Australia. They attempted to both quantify their workload and assess the appropriateness of their IVAB prescribing. Over a 12-month period, they managed 251 infective episodes with a median of 7 days of IVABs delivered within their service. They suggest that there were 3084 days where OPAT replaced inpatient care, associated with an impressive cost saving of \$A1.82 million in a single year. Ensuring that children receive the same quality of care at home as they would have received in hospital is paramount, and the authors report low complication rates: $11 \%$ of patients experienced adverse events and only 4\% of patients required an unplanned

Correspondence to Dr Sanjay Valabh Patel, Department of Paediatric Infectious Diseases and Immunology, University Hospital Southampton NHS Foundation Trust, Southampton General Hospital, Tremona Road, Southampton S016 6YD, UK; sanjay.patel@uhs.nhs.uk readmission. Although all the adverse events were related to vascular access as opposed to side effects from their medication, the overall rate of central lineassociated blood stream infections was only $0.9 \%$. This is far lower than described in previous studies.

These numbers at first glance appear very impressive. However, on closer inspection, one gets the impression that the dataset is perhaps incomplete, given that the information on adverse events is only available for 133 of the 251 episodes they managed. Robust data collection, including feedback from patients and parents, is a central tenet of an effective OPAT service; without outcome information, quality of care and cost-effectiveness cannot be robustly ascertained. In addition, it is hard to evaluate what these figures really mean without denominator or comparator data. Standardising data collection across hospitals enables centres to benchmark themselves against other paediatric OPAT services. The patient management system and registry developed by the BSAC OPAT initiative offers such a resource (http://e-opat.com/ opat-patient-management-system/).

Prospective data collection also offers potential benefits beyond patient safety. There are mounting concerns about the evolution of antimicrobial resistance and monitoring the judicious use of antimicrobials to preserve their future effectiveness is essential. The convenience of OPAT makes it extremely easy to administer long courses of IVABs. Due to the paucity of high-quality evidence available to inform decision making about antibiotic prescribing, including the optimal route and duration of administration, Hodgeson et $a l^{3}$ have been forced to resort to expert opinion to decide whether prescribing was appropriate or not within their cohort of patients. In their opinion, $8 \%$ of IVAB courses were too long and 6\% too broad, though infectious diseases specialists are often more likely to err on the side of caution and prescribe longer courses of antibiotics than other doctors. ${ }^{4}$ Data collected from OPAT services comparing antibiotic prescribing habits and outcomes offers a solution to this dearth of evidence and the demonstration of efficacy of shorter courses could also help rationalise treatment.

The majority of children requiring treatment with IVABs are managed in local hospitals and require short antibiotic courses. Such ambulatory OPAT services face different challenges to tertiary OPAT services. Ensuring robust clinical governance and routine data collection is complicated by different teams in different parts of the hospital being involved in the management of these patients. There is also considerable variation between clinicians in deciding if and when a child is suitable for ambulatory OPAT. In addition, the safety of admission avoidance strategies (ie, sending a child home directly without admission) needs to be established, although the fact that $18 \%$ of the cohort described by Hodgson et $a l^{3}$ were discharged directly from the emergency department is reassuring. The majority of these patients had skin/soft tissue infections or urinary tract infections. Other studies have shown that children presenting with pre-septal cellulitis, lymphadenitis and young infants with fever can also be managed using a similar admission avoidance strategy. ${ }^{5}$ Only through routine data collection on this cohort of patients, along with qualitative research into the opinions of parents and clinicians, will we be able to identify the potential barriers against the introduction of ambulatory OPAT programmes in all hospitals managing children. Hodgson et $a l^{3}$ have demonstrated that tertiary OPAT can be safely delivered and this should provide further impetus for introducing them in all regional children's hospitals. What is now urgently needed is more data on the appropriateness and effectiveness of ambulatory OPAT and implementation strategies for ensuring robust clinical governance and effective data collection. This may prove more challenging!

Competing interests None declared.

Provenance and peer review Commissioned; internally peer reviewed.

To cite Patel SV. Arch Dis Child 2016;101:873-874.

Received 3 June 2016

Revised 26 June 2016

Accepted 27 June 2016

Published Online First 25 July 2016

S Linked

http://dx.doi.org/10.1136/archdischild-2015-309731

Arch Dis Child 2016;101:873-874.

doi:10.1136/archdischild-2016-311099 


\section{Editorials}

\section{REFERENCES}

1 Rucker RW, Harrison GM. Outpatient intravenous medications in the management of cystic fibrosis. Pediatrics 1974;54:358-60.

2 Patel S, Abrahamson E, Goldring S, et al. Good practice recommendations for paediatric outpatient parenteral antibiotic therapy ( $p$-OPAT) in the UK: a consensus statement. J Antimicrob Chemother 2015;70: 360-73.

3 Hodgson KA, Huynh J, Ibrahim LF, et al. The use, appropriateness and outcomes of outpatient parenteral antimicrobial therapy. Arch Dis Child 2016;101:886-93.

4 Daneman N, Shore K, Pinto R, et al. Antibiotic treatment duration for bloodstream infections in critically ill patients: a national survey of Canadian infectious diseases and critical care specialists. Int J Antimicrob Agents 2011;38:480-5.

5 Brugha RE, Abrahamson E. Ambulatory intravenous antibiotic therapy for children with preseptal cellulitis. Pediatr Emerg Care 2012;28:226-8. 